\title{
Borderline personality disorder - a misunderstood disorder
}

S Rao

Personality disorders impact up to $6 \%$ of people in the community (1). Of these, borderline personality disorder (BPD) is the most commonly diagnosed. It is also among the most severe of all personality disorders (2). Studies conducted in Western countries report that BPD impacts at least $1 \%$ of the population (3-5). The representation of BPD increases in clinical settings, affecting $15 \%$ to $20 \%$ of patients in mental health services and $6 \%$ of patients in primary care (6-9). Despite its high prevalence, BPD remains a highly stigmatised and misunderstood disorder. This is encapsulated in a quote from Professor John Gunderson, a pioneer and visionary in the field, who once stated that "BPD is to psychiatry, what psychiatry is to medicine" (10).

BPD is characterised by instability in mood, relationships and self-identity, together with impulsivity, fear of abandonment, anger dyscontrol, recurrent suicidal and self-injurious behaviours, episodes of dissociation and micro-psychosis. Some patients with BPD may experience severe and persistent self-loathing, chronic hopeless, emptiness and "identity-less-ness" (11). BPD is a complex mental illness with significant morbidity and mortality. About $85 \%$ of patients self-injure. People with BPD are chronically suicidal; the suicide rate for BPD is $10 \%$ (12). When their illness is active, people with BPD endure miserable and painful lives. Chronic suicidality appears to be used as a coping strategy. People with BPD often experience life to be so painful that maintaining suicidal ideations helps them to stay alive (13). BPD contributes to about $95 \%$ of all personality disorder-related suicides (14).

BPD has a wide impact, causing significant distress to patients, families and friends. Clinicians working to support people with BPD may also experience distress and significant emotional reactions. BPD tends to cooccur with other psychiatric disorders such as depression, post-traumatic stress disorder, eating disorders and substance use disorders. The presence of BPD negatively impacts the prognosis of co-occurring psychiatric disorders, particularly depressive disorders (15). Co-occurring psychiatric disorders respond less well when treated separately in the absence of treatment of BPD with specific psychotherapies. Certain medical illnesses also tend to co-occur with BPD, with a third of
BPD patients having chronic pain conditions (16) while a third of women with BPD have polycystic ovarian syndrome (17). The prevalence of metabolic syndrome, obesity and chronic fatigue syndrome is also significant. Overall, the life span of a person with BPD may be reduced by up to 20 years (18).

The study of BPD has attracted significant global interest during the last three decades, resulting in a vast body of research. As a result, several misconceptions about BPD have been corrected. One of these is the myth that BPD is a disorder of women; it is now clear that BPD impacts both genders equally (3).

BPD impacts people across their life span. Although BPD usually emerges during adolescence (19), it is often not diagnosed until the age of 18 . Therefore, opportunities for early intervention are often missed. Recognising the need for early diagnosis and intervention, the Australian National Health and Medical Research Council (NHMRC) clinical practice guideline for the management of BPD has recommended that BPD be diagnosed from the age of 12 (20). Some recent reports have noted that BPD symptoms can persist beyond 65 years of age or manifest for the first time in later life (21).

Recent studies have improved our understanding of the etiology of BPD. The genetic heritability of BPD is believed to be about $50 \%$ (22). This impacts the expression of brain mechanisms that are involved in emotion regulation. Biological abnormalities such as hyperactive amygdala, poor executive control of the amygdala via prefrontal cortical pathways, anterior insula abnormality (causing profound incapacity to co-operate) have all been reported. In the past, childhood trauma was considered to be a causative factor in the development of BPD. Although trauma is very common in BPD, it appears to be a risk factor rather than being essential for the development of BPD. Complex interactions between biological vulnerabilities and environmental factors (e.g. trauma, attachment disorders, bullying, invalidating interpersonal experiences during developmental years) may result in individual patients carving their own unique pathways towards the development of BPD. The Australian NHMRC Guideline (2012) concluded that 'having BPD is not the person's own fault - it is a condition of the brain and mind' (20). 
The diagnosis of BPD was previously thought to have a poor prognosis; clinicians held a pessimistic view about its treatment and clinical outcomes. Clinicians often hesitated to give patients a diagnosis of BPD, fearing the stigma and discrimination that patients would experience. When clinicians did make this diagnosis, they often avoided discussing this with their patients because of its pejorative name, perceived lack of treatment and poor prognosis.

Two key longitudinal follow-up studies, the Collaborative Longitudinal Personality Disorders Study (23) and the McLean Study of Adult Development (24) have dispelled the belief that the prognosis for BPD is poor. The prognosis is optimistic for patients with BPD; the evidence tells us that most people with BPD will eventually achieve a life worth living, find their place in the world and stop wanting to kill themselves (23). Most patients can achieve symptomatic remission when appropriate psychological treatments are provided. However, recovery (having at least one meaningful relationship and being able to work) may take longer and be more difficult to achieve. Once remission is achieved, relapse rates are as low as $15 \%$ (18).

Psychotherapy is the treatment of choice for BPD. As yet, no psychotropic medications have been patented or indicated for the treatment of BPD despite their widespread use for treating BPD symptoms. Psychotropics may at best be used for short-term, time limited crisis management or as adjuncts to psychotherapies. There is a wide range of evidence-based psychological treatments for BPD, however the "big four" remain the most popular. The big four treatments include Dialectical Behaviour Therapy (DBT); (25), Mentalization Based Treatment (MBT), Schema Focused Therapy (SFT) and Transference Focused Therapy (TFP). These are highly specialized BPD-specific treatments that can help patients achieve remission from BPD. Although these highly specialized treatments are effective, they are expensive to deliver and clinicians providing these treatments require extensive and intense training. Therefore, these treatments are less suitable for a national population health approach. However studies have demonstrated that BPD-specific generalist treatments such as General Psychiatric Management, Structured Clinical Management, and Good Clinical Care are as effective as the specialist treatments (26). Research has highlighted the commonalities among specialist and generalist treatments, leading to the development of integrated, common factors based, stepped-care treatments that can be adapted to local mental health systems (26).

\section{BPD in the context of Eastern cultures:}

There is paucity of definitive epidemiological data about the prevalence of BPD in Eastern countries. However, interest in the detection and treatment of BPD is gathering momentum in these countries as the impact of untreated BPD is recognised. The stigma around BPD is likely to be significant in many Eastern cultures given the prevalent stigma around suicide and self-injury, hallmark features of BPD. In countries where mental health care is not widely accessible, treatment of BPD is likely to pose a big challenge. Most of the evidence-based psychological treatments for BPD were developed in Western countries for Western populations in the context of individualistic cultures. It is not clear whether these treatments are the best options for Eastern countries, where collectivistic cultures are common.

Veerasamy et al., (27) suggested that the focus in developing countries should start with collecting epidemiological data related to BPD and then improve service delivery, create more awareness, identify and diagnose patients with BPD, develop treatment guidelines, and train clinicians in psychological therapies. Looking to the future, prevalence studies need to be undertaken to establish how BPD may manifest in Eastern countries. The symptom profile of BPD may turn out be somewhat different, reflecting cultural differences. Treatments such as DBT and Acceptance and Commitment Therapy that have roots in Buddhist mindfulness traditions may render themselves more adaptable to Eastern contexts. Psychological interventions that actively include family members alongside the person experiencing BPD and have been adapted to local cultural contexts will need to be developed and tested.

\section{Acknowledgements}

I wish to thank my colleagues A/Prof Jillian Broadbear and Parvaneh Heidari for their helpful feedback and assistance while preparing this manuscript.

\section{Disclosure statement}

None conflicts of interest to declare.

R Rao, Clinical Director, Spectrum, Centre of Clinical Excellence for Personality Disorders, Melbourne, Australia.

Email: Sathya.Rao@easternhealth.org.au

https://orcid.org/0000-0001-9364-8831

\section{References}

1. Huang Y, Kotov R, de Girolamo G, Preti A, Angermeyer $\mathrm{M}$, Benjet $\mathrm{C}$, et al. DSM-IV personality disorders in the WHO World Mental Health Surveys. The British Journal of Psychiatry: the Journal of Mental Science. 2009; 195(1): 46. 
2. Chanen A, McCutcheon L, Jovev M, Jackson H, McGorry P. Prevention and early intervention for borderline personality disorder. Medical Journal of Australia. 2007; 187(7): S18-21.

3. Grant BF, Chou SP, Goldstein RB, Huang B, Stinson FS, Saha TD, et al. Prevalence, correlates, disability, and comorbidity of DSM-IV borderline personality disorder: results from the Wave 2 National Epidemiologic Survey on Alcohol and Related Conditions. The Journal of clinical Psychiatry. 2008; 69(4): 533.

4. Trull TJ, Jahng S, Tomko RL, Wood PK, Sher KJ. Revised NESARC personality disorder diagnoses: gender, prevalence, and comorbidity with substance dependence disorders. Journal of Personality Disorders. 2010; 24(4): 412-26.

5. Torgersen S, Myers J, Reichborn-Kjennerud T, Roysamb E, Kubarych TS, Kendler KS. The heritability of Cluster B personality disorders assessed both by personal interview and questionnaire. Journal of Personality Disorders. 2012; 26(6): 848-66.

6. Kass F, Skodol AE, Charles E, Spitzer RL, Williams JB. Scaled ratings of DSM-III personality disorders. The American Journal of Psychiatry. 1985; 142(5): 627.

7. Zimmerman M, Rothschild L, Chelminski I. The Prevalence of DSM-IV Personality Disorders in Psychiatric Outpatients. The American Journal of Psychiatry. 2005: 1911-8.

8. Korzekwa MI, Dell PF, Links PS, Thabane L, Webb SP. Estimating the prevalence of borderline personality disorder in psychiatric outpatients using a two-phase procedure. Comprehensive Psychiatry. 2008; 49(4): 380-6.

9. Gross R, Olfson M, Gameroff M, Shea S, Feder A, Fuentes $\mathrm{M}$, et al. Borderline personality disorder in primary care. Archives of Internal Medicine. 2002; 162(1): 53-60.

10. Gunderson JG. Borderline personality disorder: ontogeny of a diagnosis. The American Journal of Psychiatry. 2009; 166(5): 535

11. Zanarini MC, Frankenburg FR, DeLuca CJ, Hennen J, Khera GS, Gunderson JG. The pain of being borderline: dysphoric states specific to borderline personality disorder. Harvard Review of Psychiatry. 1998; 6(4): 201-7.

12. Paris J, Zweig-Frank H. A 27-year follow-up of patients with borderline personality disorder. Comprehensive Psychiatry. 2001; 42(6): 482-7.

13. Paris J. Half in love with death: Managing the chronically suicidal patient: Routledge; 2007.

14. Rao S, Bugeja L, Dwyer J, Broadbear JH, editors. Identification of Opportunities for Intervention Prior to Death By Suicide in People With Borderline Personality Disorder. RANZCP Congress 2019; 2019; Cairns,
Australia: Australian \& New Zealand Journal of Psychiatry.

15. Skodol AE, Grilo CM, Keyes KM, Geier T, Grant BF, Hasin DS. Relationship of Personality Disorders to the Course of Major Depressive Disorder in a Nationally Representative Sample. American Journal of Psychiatry. 2011; 168(3): 257-64.

16. Sansone RA, Sansone LA. Chronic pain syndromes and borderline personality. Innovations in clinical neuroscience. 2012; 9(1): 10 .

17. Roepke S, Ziegenhorn A, Kronsbein J, Merkl A, Bahri S, Lange J, et al. Incidence of polycystic ovaries and androgen serum levels in women with borderline personality disorder. J Psychiatr Res. 2010; 44(13): 847-52.

18. Chesney E, Goodwin GM, Fazel S. Risks of all-cause and suicide mortality in mental disorders: a meta-review. World Psychiatry : official journal of the World Psychiatric Association. 2014; 13(2): 153-60.

19. Chanen MA, McCutcheon KL, Germano JD, Nistico DH, Jackson DH, McGorry DP. The HYPE Clinic: An Early Intervention Service for Borderline Personality Disorder. Journal of Psychiatric Practice. 2009; 15(3): 163-72.

20. National Health Medical Research Council. Clinical practice guideline for the management of borderline personality disorder. Melbourne: National Health and Medical Research Council 2012.

21. Beatson J, Broadbear JH, Sivakumaran H, George K, Kotler E, Moss F, et al. Missed diagnosis: The emerging crisis of borderline personality disorder in older people. London, England 2016; 1139-45.

22. Torgersen S, Lygren S, Oien PA, Skre I, Onstad S, Edvardsen J, et al. A twin study of personality disorders. Comprehensive Psychiatry. 2000; 41(6): 416-25.

23. Gunderson JG, Stout RL, McGlashan TH, Shea MT, Morey LC, Grilo CM, et al. Ten-year course of borderline personality disorder: psychopathology and function from the Collaborative Longitudinal Personality Disorders study. Arch Gen Psychiatry. 2011; 68(8): 827-37.

24. Zanarini MC, Frankenburg FR, Reich DB, Wedig MM, Conkey LC, Fitzmaurice GM. Prediction of time-toattainment of recovery for borderline patients followed prospectively for 16 years. Acta Psychiatr Scand. 2014; 130(3): 205-13.

25. Linehan MM. Dialectical behavioral therapy: A cognitive behavioral approach to parasuicide. J Personal Disord. 1987; 1(4): 328-33.

26. Bateman AW, Krawitz R. Borderline personality disorder: an evidence-based guide for generalist mental health professionals: Oxford University Press; 2013.

27. Veerasamy K, Guruvaiah L. Overview of the treatment for borderline personality. Sri Lanka Journal of Psychiatry. 2013; 4(2). 